\title{
I film associativi autobiografici dei migranti e lo spazio di ricezione dei foyers parigini
}

Les films associatifs autobiographiques de migrants et l'espace d'accueil des foyers parisiens

\section{Guglielmo Scafirimuto}

\section{QpenEdition Journals}

\section{Edizione digitale}

URL: http://journals.openedition.org/ctd/1415

DOI: $10.4000 /$ ctd. 1415

ISSN: 2491-1437

\section{Editore}

Chaire Unesco Pratiques émergentes en technologies et communication pour le développement

\section{Edizione cartacea}

ISBN: 2491-1437

Notizia bibliografica digitale

Guglielmo Scafirimuto, «I film associativi autobiografici dei migranti e lo spazio di ricezione dei foyers parigini », Communication, technologies et développement [En ligne], 7 | 2019, mis en ligne le 20 juin 2019, consulté le 05 juillet 2019. URL : http://journals.openedition.org/ctd/1415 ; DOI : 10.4000/ ctd. 1415

Questo documento è stato generato automaticamente il 6 luglio 2019

Communication, technologies et développement 


\section{I film associativi autobiografici dei migranti e lo spazio di ricezione dei foyers parigini}

Les films associatifs autobiographiques de migrants et l'espace d'accueil des foyers parisiens

Guglielmo Scafirimuto

الافلام الجمعياتية الخاصة بالسيرة الذاتية للمهاجرين ومنطقة استقبال الأسر الباريسية

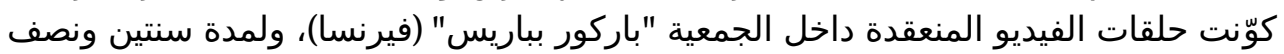

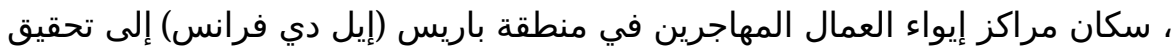
منتجات سمعية بصرية.

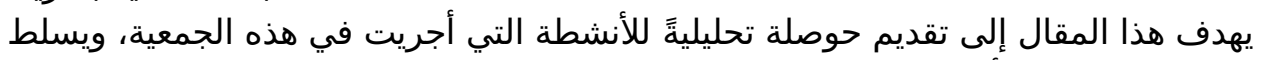

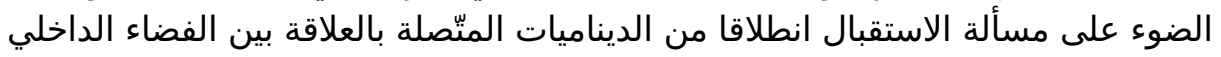

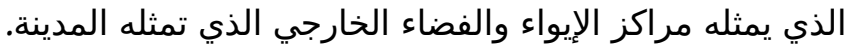

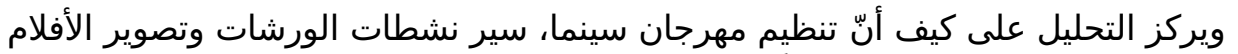

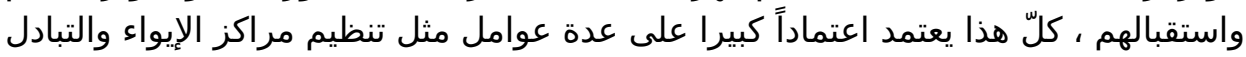

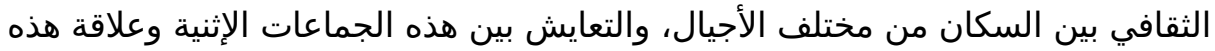

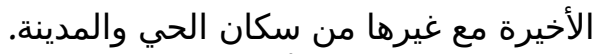

تقدم الأفلام القصيرة للجمعية كخلط بين الفيلم الوثائقي والخيالي، على أساس مناس منهج تشئ تشاركي

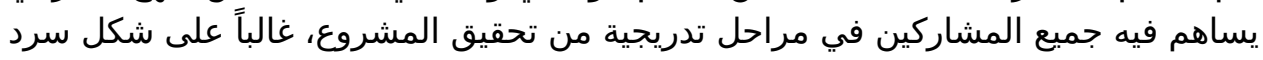
قصص ذاتية للمهاجرين بدون والبا علي شائق.

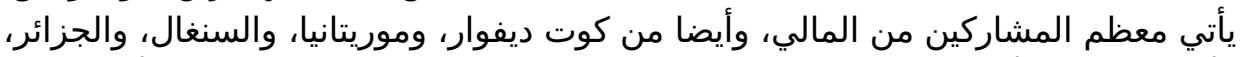

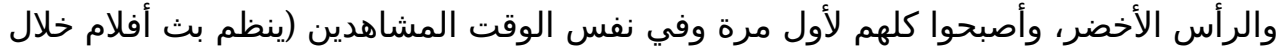

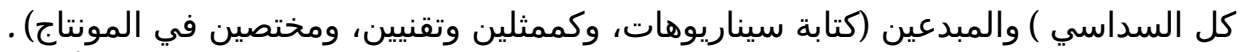

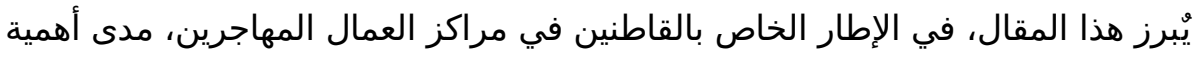

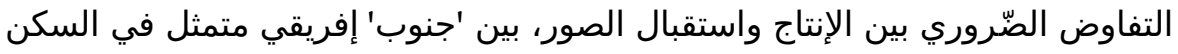

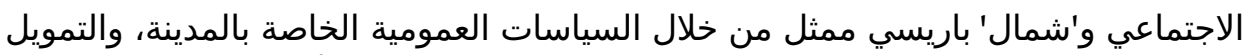
العمومي وأماكن النشر والمناقشة والموية مراكز، باريس، الهجرة الأفريقية، فيديو، تشاركية، العلوم التربوية، عرض الشوالمن 
1 Essendo il tema della "ricezione" il filo conduttore di questo corpus di interventi, tengo a precisare fin da subito che la ricezione del presente testo, il quale sarà in forma di resoconto analitico, deve tener conto di un duplice modo auto-riflessivo: una critica autobiografica sullo sforzo di mettere in atto una narrazione autobiografica da parte di individui che hanno da poco emigrato. Il seguente articolo dimostrerà che le riflessioni sulla pedagogia e sull'espressione delle minoranze - dare spazio, modo, voce e forma all'autorappresentazione - non possono che articolarsi all'interno di determinati contesti e condotte al centro di negoziazioni tra attori ed esigenze diverse. Mi focalizzerò qui su un'azione particolare che conduco personalmente da due anni in ambito associativo - gli Ateliers Vidéo - su un terreno, anch'esso peculiare, che è quello delle residenze sociali francesi per i migranti, chiamate "foyers des travailleurs migrants". Il testo tenterà dunque di elucidare, tramite la questione della ricezione delle immagini, il ruolo della mia associazione culturale nel rapporto tra le politiche pubbliche d'integrazione dei migranti nei quartieri cosiddetti “politici” della città, e le pratiche che regolano i suddetti foyers. Laddove la produzione di narrazioni autobiografiche si incontra e a volte si scontra con le aspettative dei diversi luoghi di diffusione e dei suoi pubblici.

\section{I foyers e l'intervento associativo}

2 Con la formula "foyers des travailleurs migrants", mi riferisco a un insieme molto vasto di alloggi sociali nati in tutta la Francia e specialmente in Ile-de-France negli anni cinquanta per essere destinati alle popolazioni immigrate (maschili) africane che partivano dalle colonie francesi al fine di trovare un lavoro nella regione parigina. Furono innanzitutto gli Algerini che, emigrando sulle scie della Guerra d'Algeria, vennero considerati all'epoca come lavoratori a breve termine e fecero l'oggetto di questa politica abitativa legata al controllo coloniale. Al Nord Africa seguirono poi le migrazioni postcoloniali dell'Africa Occidentale. Vennero costruite allora delle residenze ai margini delle principali città francesi, nei quartieri periferici o direttamente in banlieue, dove i residenti cominciarono a vivere isolati e in condizioni insalubri, occupando in tanti una stessa stanza e condividendo una cucina per tutto il foyer. Malgrado gli sforzi recenti volti a riconvertire certi foyers più datati in uno stile più moderno d'alloggio a vocazione sociale, il rapporto tra i residenti del foyer e gli altri abitanti del quartiere, come anche del resto della città, resta praticamente inesistente. Ancora oggi, i foyers costituiscono degli spazi invisibili e sconosciuti per la maggior parte dei parigini, un terreno privilegiato per osservare le tensioni tra le comunità diasporiche di lunga data e le politiche comunali. In effetti, a causa dei raggruppamenti di villaggi interi africani (della Cabilia algerina o della regione di Kayes in Mali, per citare due maggiori esempi), dei conflitti permanenti con i gestori e della forte presenza degli anziani residenti ormai in età senile, $i$ foyers rappresentano, più che un alloggio "provvisorio" com'era previsto dal Ministero, delle residenze a lungo termine che tendono a riprodurre i sistemi comunitari e le pratiche legate alle società d'origine. Gestiti internamente dai délégués che si occupano dell'amministrazione, dagli imam che regolano la vita religiosa e dagli anziani che controllano i più giovani, i foyers presentano un funzionamento molto oliato e gerarchico, spesso chiuso su se stesso. Le nuove generazioni che arrivano, precarizzate dalla disoccupazione e da tutte le difficoltà dovute al fatto di essere sans papiers, trovano in loco il vantaggio di un aiuto di un residente proveniente dalla stessa famiglia o dallo stesso villaggio, ma, finché restano nel 
foyer, devono anche far fronte la maggior parte delle volte a una immobilità sociale, linguistica e culturale sicuramente più rilevante che in altri contesti.

3 Aldilà del dibattito sulla legittimità e l'efficacia di questo tipo di politica abitativa, mi sembra in ogni caso da sottolineare la scelta dei municipi degli arrondissements parigini implicati di appoggiare i progetti culturali associativi che da tanto tempo organizzano in questi "quartiers politiques" degli ateliers di lingua, di teatro, d'informatica, di cinema etc. per i pubblici marginalizzati. La speranza delle istituzioni francesi riguardo l'animazione culturale nei foyers è quella di rompere la «diffidenza reciproca tra i cittadini e i lavoratori migranti »: "L'obiettivo è di far conoscere meglio nella città le culture delle comunità straniere residenti e di incitare quest'ultime a partecipare alle manifestazioni culturali locali $»^{1}$ Grazie a dei finanziamenti pubblici dei suddetti municipi - soprattutto del $20^{\mathrm{e}}, 13^{\mathrm{e}}$ e $14^{\mathrm{e}}$ arrondissement - e della DRAC (Direction Régionale des affaires culturelles d'Ile-de-France), la mia associazione, chiamatasi all'inizio Attention Chantier e adesso Parcours, ha potuto intrattenere dal 2008 delle relazioni durature e prolifiche con i foyers interessati (Bisson a Belleville, Tolbiac a Olympiades, Gergovie a Plaisance) e con altri attori associativi similari (Aarao, Raconte nous ton histoire, Autremonde, etc.). La sfida è sempre stata quella di sperimentare e di rinnovare il suo ruolo di mediatrice tra lo spazio interno del foyer e lo spazio esterno del quartiere. Da un lato, l'apertura dei foyers passa dalla sua trasformazione in un bacino di ricezione cinematografica : ogni estate, un festival di cinema è stato organizzato nel circuito più largo possibile dei foyers migranti parigini per animare delle proiezioni di film seguite da cene collettive, concerti e dibattiti. Dall'altro lato, la partecipazione attiva dei residenti alla vita cittadina passa dalla presa di parola favorita dalla creazione di una radio partecipativa e dai diversi ateliers (stop-motion, pittura, video).

\section{Le azioni dell'associazione e le dinamiche di ricezione}

Intendo dunque iniziare con la prima delle attività dell'associazione, in modo da accentuare la dimensione della ricezione all'interno dei foyer in occasione dei festival di cinema e dei cine-foyers (delle proiezioni saltuarie di film nei foyers, normalmente in forma collaborativa con altre associazioni). Bisogna innanzitutto ricordare che i residenti dei foyers non hanno generalmente modo di accedere alle sale cinematografiche parigine, troppo care e spesso lontane dal loro circuito d'azione abituale, né l'hanno avuto prima nel loro paese d'origine, in ragione dell'assenza di cinema nei villaggi da cui vengono, o anche nelle città dove hanno certuni vissuto o da dove sono passati. Le proiezioni, trasformando il foyer in uno spazio di ricezione, permettono in questo modo ai residenti di diventare per la prima volta spettatori d'un film sul grande schermo. Consentono inoltre di dedicarsi all'istallazione del materiale, di condividere un momento conviviale seduti sui tappeti africani disposti di fronte lo schermo, di partecipare ai pasti comuni e ai dibattiti nonché d'incontrare persone esterne al foyer, essendo il festival aperto a tutti. La scelta dei film - documentari o di finzione, e anche animazione -, così come delle decorazioni, delle musiche, del mangiare e delle tematiche in discussione, tutto verte sui paesi d'origine dei residenti, quindi sull'Africa Occidentale, in maniera tale da attirare all'evento tutte le generazioni presenti (in generale gli anziani restano abituati ai piatti africani e i giovani continuano a preferire l'hip-hop e il reggae al rock o al pop per esempio) e per apportare ugualmente dei punti vista innovativi sulle questioni attuali associate all'Africa e alle migrazioni recenti. I film programmati sono tutti o in francese o 
nelle lingue più diffuse nel foyer - bambara e soninke - sottotitolate in francese, per favorire la comprensione dell'intero pubblico. L'evento annuale del festival è stato sempre ben accolto dai partecipanti, poiché queste residenze mancano di momenti ricreativi al livello della collettività. Le proiezioni diventano così un'occasione festiva per il foyer e un volantinaggio comprendente a volte una pubblicità porta a porta nelle stanze è sollecitato per alimentare una maggiore affluenza. Altre associazioni o delle personalità legate alle questioni dibattute sono invitate inoltre al festival in vista di un dialogo più nutrito con il mondo esterno al foyer. Le proiezioni sono organizzate generalmente la sera in modo da evitare l'illuminazione naturale e hanno luogo in una corte all'aperto (se il foyer ne dispone) o in una grande sala polivalente che è sempre presente all'interno. Durante la visione del film, il pubblico è abitualmente abbastanza rumoroso, poiché gli spettatori possono arrivare e partire liberamente in qualsiasi momento. La maggioranza del pubblico reagisce alle sequenze viste sullo schermo con commenti a voce alta o con risate nei punti più comici, la partecipazione essendo nettamente più visibile e intensa in questo contesto che in quello della regolare sala cinematografica. Svolgendosi in un ambiente totalmente musulmano, le proiezioni si adattano ovviamente al Ramadan in termini di periodo dell'anno e alle cinque preghiere giornaliere per quel che riguarda gli orari. Tutti questi fattori contribuiscono allora all'impressione di essere proprio in uno spazio d'entre-deux, in cui tutto rinvia all'Africa rimanendo pur sempre a Parigi. Questa à anche una delle ragioni che attira non soltanto il pubblico dei residenti dei foyers, ma anche chi viene per la prima volta dall'esterno e che finisce per apprezzare le condizioni inedite di questa esperienza di ricezione filmica.

Figura 1. Festival al foyer Gergovie (Luglio 2017)

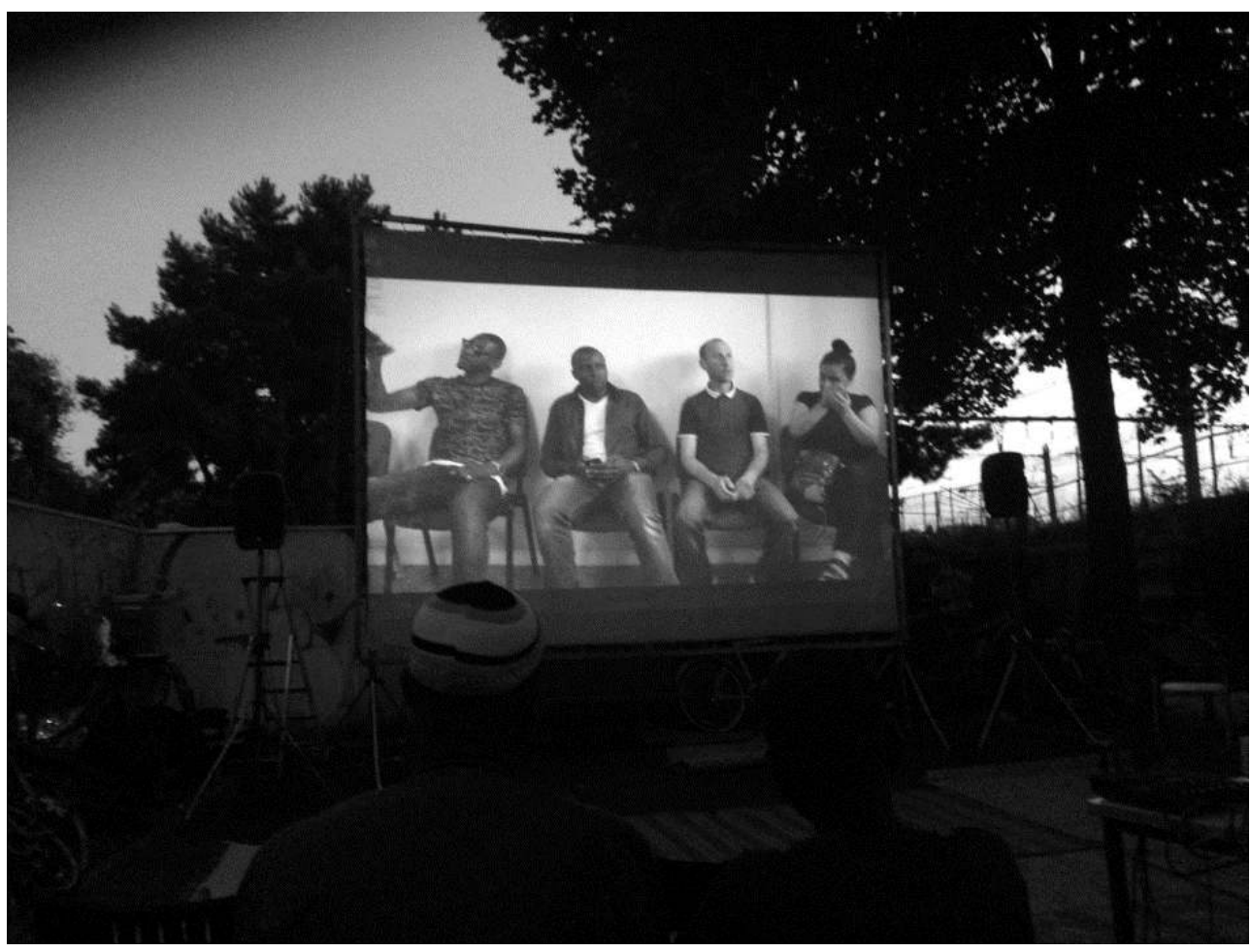

5 A questo punto vorrei passare all'approfondimento della seconda parte del progetto: i film associativi autobiografici realizzati nell'ambito degli Ateliers Vidéo, che coordino dall'inizio del 2016. Gli Ateliers Vidéo si svolgono in due foyers alla volta con due gruppi 
paralleli di una decina di partecipanti che si riuniscono ciascuno un giorno alla settimana per due ore. I partecipanti si impegnano (anche se in concreto la natura di questo impegno è sempre problematicamente altalenante o solo temporanea) sulla continuità di un anno diviso in due semestri, corrispondenti alla creazione totale di due film per ogni gruppo. Gli obiettivi principali degli ateliers sono prima di tutto: sensibilizzare il pubblico dei foyers, normalmente ai margini della vita culturale e sociale della città, al linguaggio cinematografico e all'interesse di produrre e diffondere in prima persona delle immagini ; iniziare i partecipanti alle diverse tappe della realizzazione audiovisiva, dalla pre-produzione alla post-produzione. Si tratta di un lavoro partecipativo che pone al centro delle sue preoccupazioni e della sua metodologia una sorte di "pedagogia militante", basata sul concetto di autorappresentazione delle minoranze e sull'intervento attivo di nuovi attori sociali tramite la messa a disposizione degli strumenti di espressione. La realizzazione di cortometraggi permette ai partecipanti di prendere la parola attraverso l'immaginazione, appropriandosi dei mezzi tecnici e creativi atti a comunicare la loro esperienza migratoria (o semplicemente autobiografica) a partire da un punto di vista soggettivo. I partecipanti sono invitati a costruire insieme le narrazioni filmiche in maniera da poter unire l'apprendimento pratico alla riflessione personale sul loro vissuto. Ognuno di loro può scegliere poi se e quando essere sceneggiatore, regista, fonico, attore e/o montatore.

Da che contesto e basi nasce questa che chiamo "pedagogia militante" degli ateliers? Il progetto si iscrive certo nell'ambito nazionale francese della pedagogia audiovisiva e dell'educazione popolare, recentemente coordinata dal gruppo Passeurs d'images. Tuttavia, gli Ateliers Vidéo, più che con il versante istituzionale concepito come educazione verso il "popolo", si allineano con le azioni solidari militanti insieme al "popolo", promulgate dalla rete associativa parigina: i collettivi dei sans papiers, Paris d'exil, Atelier des artistes en exil, BAAM, Raconte nous ton histoire, Aarao, Belleville en Vues, Belleville Citoyenne, CIP20, Autre Monde, Canal Marche, etc. A proposito dell'insistenza del lavoro sociale e delle politiche cittadine verso l'integrazione, l'educazione e la riqualificazione, Boucher parla infatti di « dinamiche di ricomposizione del controllo sociale $»^{2}$ Delicata (e forse ipocrita) distinzione - tra istituzionale e militante - che però a mio avviso aiuta a delineare un orizzonte attitudinale diverso : concepire i partecipanti (e non "allievi" o "utenti") non come oggetti di "ricezione" di una politica sociale, ma come protagonisti anch'essi di un cambiamento e di un'azione comune. Ovviamente mi rendo perfettamente conto dell'ambivalenza di quest'aspirazione (le associazioni sono comunque finanziate dagli enti pubblici) e dell'idealismo del modello partecipativo, che deve confrontarsi a una pratica sul terreno che presenta sempre numerose variabili, imprevisti e situazioni d' impasse. Sicuramente alla base del mio impegno associativo vi sono le mie ricerche universitarie estetiche e antropologiche sull'autorappresentazione cinematografica dei migranti, sui Postcolonial Studies, sull'esempio italiano dell'associazione Asinitas, che ha portato diversi giovani anche appena sbarcati alla realizzazione di film. Le letture sulla mediazione culturale hanno apportato anche qualche spunto metodologico. Gisèle Legault per esempio indica quelli che per lei sono i presupposti chiave di un'azione interculturale : il sapere (savoir : informarsi), il saper-essere (savoir-être : avere coscienza del proprio ruolo), il saper-fare (savoir-faire: rendere l'intervento compatibile col contesto) ${ }^{3}$ In ogni caso, se i dibattiti sulla "giusta distanza" ${ }^{4} \mathrm{o}$ sulla "prossimità relativa" 5 da intraprendere in ogni rapporto con l'Altro sembrano non sfociare in un punto d'incontro, si può essere d'accordo che la comunicazione interculturale, seppur un "dilemma", è comunque "una necessità sociale", come lo ricorda Said Bouamama ${ }^{7} \mathrm{La}$ 
"pedagogia militante" nasce proprio da una necessità : la politica delle azioni sociali è ancora indispensabile e strutturata dall' "alto", ma deve mirare a un cambiamento concreto per chi continuerà queste iniziative dal "basso".

Figura 2. Atelier Vidéo al foyer Gergovie (Gennaio 2018)

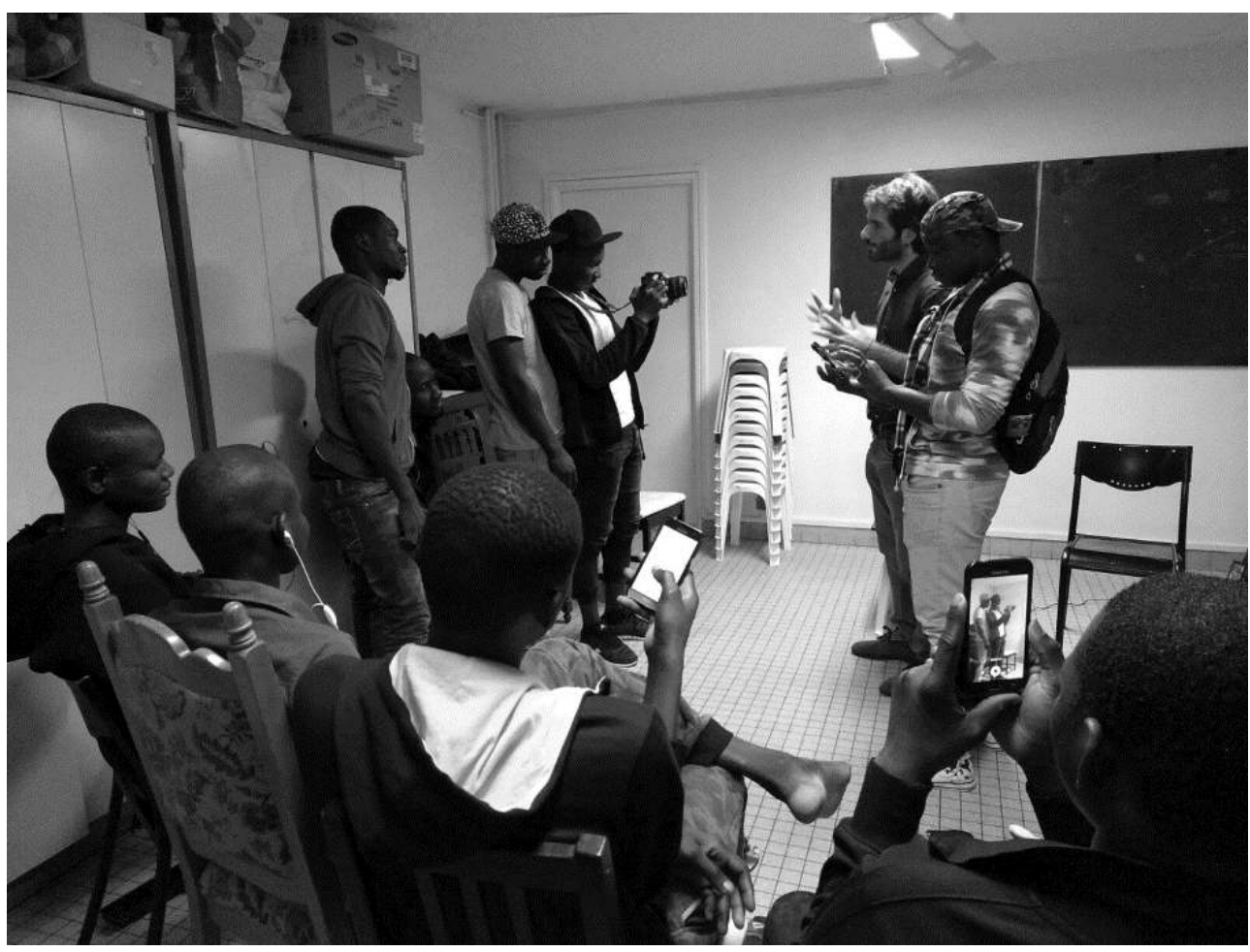

\section{Gli Ateliers Vidéo : creare spazi di produzione e di ricezione}

7 L'artista multimedia Krzystof Wodiczko, che ha lavorato dagli anni novanta con i migranti, specifica la priorità del "pubblico interno" (inner public) - le persone coinvolte in tutte le tappe del progetto partecipativo - rispetto al "pubblico esterno" (outer public), che è solo fruitore del lavoro finale dei partecipanti ${ }^{8}$ Il ruolo dell'artista (e del coordinatore nel mio caso) è secondo Wodiczko quello di essere un "catalizzatore" $\mathrm{di}$ questo "pubblico interno", tramite il metodo socratico della maieutica, che aiuta a far nascere spontaneamente l'ispirazione artistica altrui. Queste riflessioni mi hanno decisamente suggerito certe posture, ma qui di seguito riporto la loro negoziazione con il contesto specifico dei foyers parigini.

8 Al fine di comprendere al meglio il contesto comunicativo degli ateliers, si deve tenere in conto che quasi la totalità dei contatti dei partecipanti è presa da me tramite i corsi di lingua che sono dati nei foyers. Sono loro in seguito a decidere eventualmente se venire o meno, ma non vi è nessun appello ufficiale o convocazione volontaria concernente il cinema. Qualcosa che condiziona il buon svolgimento degli ateliers è per esempio la correlazione diretta tra la partecipazione e la ricezione : recentemente, un partecipante maliano che aveva recitato nell'ultimo documentario ha smesso di frequentare perché altri residenti, avendo visto il film in questione (avevo fatto circolare il link Vimeo sul 
nostro gruppo WhatsApp), si sono coalizzati per impedirgli di continuare quest'attività. Ciò deriva dal fatto che realizzare dei film e più in generale delle immagini, o partecipare come attore, è spesso un'attività malvista dalla comunità del foyer (gli anziani in primis, e, per trasmissione, certi giovani) e giudicata come una distrazione indecente, inutile e da evitare. I giovani sono giunti fin qui al solo fine di trovare un vero lavoro retribuito e di aiutare la propria famiglia, non per altre attività "ludiche", sostengono spesso le vecchie generazioni che si sentono responsabili delle nuove. Tuttavia questo tabù frequente dell'immagine non coinvolge sempre la maggioranza, e coloro che si sentono a disagio davanti la videocamera (per fastidio, timidezza, o per non farsi vedere dagli anziani o dai familiari) possono decidere di restare dietro, per filmare o tenere il microfono. Si può dunque affermare che i partecipanti, che perlopiù sono arrivati da poco a Parigi e non vi hanno ancora trovato dei legami sociali e affettivi, cominciano a frequentare costantemente gli ateliers più per il piacere di costruire un gruppo unito dall'amicizia che per un autentico interesse preesistente verso il mondo del cinema. Ogniqualvolta li interrogo sugli obiettivi del nostro progetto cinematografico, assisto puntualmente a una confusione generale tra il concetto di film e quello di video Youtube o di trasmissione televisiva, in un'incertezza di definizione che è rivelatrice delle loro pratiche di ricezione audiovisiva. Il punto di inizio consiste allora a cercare di spiegare i termini base che impiegherò ricorrentemente: cinema, film, cortometraggio, documentario, finzione, generi, riprese, inquadrature, etc. Ma gli ateliers non si propongono un apprendimento frontale scolastico; cerco infatti di chiarire subito che il mio ruolo in quel contesto non è quello di insegnante e autorità, ma piuttosto quello di amico e animatore. Volendo essere più ricettivo e pronto all'ascolto, uno dei primi esercizi che organizzo è quello di raccontare di fronte la videocamera - ciascuno a sua volta filma e testimonia - il proprio rapporto con il 'cinema' : che si tratti delle partite di calcio, di reportages televisivi, di clip musicali o di vecchi film americani d'azione (mi vengono citati sistematicamente Van Damme e Schwarzenegger), tutto è valido a capire le pratiche di ricezione, e dunque le conoscenze da sviluppare. La comprensione dei gusti e dei desideri di ciascuno - spesso non molto espliciti o chiari, data la debolissima esperienza da spettatori - mi aiuta a dirigere la prima fase : la formazione di una ricezione. La visione di film precedentemente realizzati dagli ateliers permette per esempio di prendere consapevolezza della possibilità concreta di riprodurre il prodotto che si è appena guardato. La ricezione diventa quindi propedeutica alla realizzazione. Posti dei riferimenti comuni -sketches, video o programmi che hanno potuto guardare - è importante dopo spostare queste prime conoscenze verso l'appropriazione dei codici della realizzazione filmica, dalla scrittura alle riprese, e infine al montaggio.

9 Durante gli esercizi a cerchio con la videocamera, la ricezione si esprime tramite la rielaborazione attiva dell'immagine attraverso l'immagine: i ragazzi, in attesa del loro turno, filmano spesso col proprio cellulare gli altri partecipanti in procinto di filmare a loro volta con la videocamera dell'associazione. Numerose immagini circolano allora al termine dei nostri incontri, che siano i suddetti video messi su Facebook dai partecipanti per mostrarli agli amici, o le foto fatte tutti assieme e condivise sul nostro gruppo Facebook e sulla nostra chat di WhatsApp. In fase di montaggio, la visione collettiva dei filmati girati suscita sempre domande, ilarità, curiosità e ulteriori dibattitti su ciò che si è detto durante le riprese (certuni sono di frequente assenti e vengono ad intermittenza). Com'è chiaro in questi casi, rivedere la propria immagine sullo schermo e la propria prima interpretazione filmata, genera imbarazzo e alcuni, discretamente, volgono altrove lo sguardo. Soprattutto al momento del sottotitolaggio (qualora gli attori abbiano 
adottato la loro lingua, comunemente il soninke o il bambara), certi partecipanti, riascoltando le loro testimonianze, esprimono il desiderio di rifilmare determinati passaggi "non detti bene". Rimontando alla fase della scrittura invece, va detto che è specialmente attraverso i dibattiti collettivi che le idee di sceneggiatura emergono e vengono successivamente fatte convergere verso una struttura narrativa. Certo, nel nostro slancio amatoriale, i codici si intrecciano volentieri. Partendo dal presupposto che sarebbe impossibile scrivere una vera sceneggiatura collettivamente (la grande maggioranza dei partecipanti è semi-analfabeta), le sequenze di dialogo sono principalmente improvvisate. I nostri film di finzione includono allora diversi elementi documentari basati sulle idee e sulle reali esperienze dei personaggi/partecipanti, una sorta di "docufiction" che finisce per costituire una delle peculiarità della nostra produzione e forse financo un abbozzo di nuovo genere filmico (con le sue regole e modalità di produzione e diffusione).

\section{Film "vietati" e film di "riparazione"}

10 A questo proposito, mi sembra pertinente citare uno degli ultimi film realizzati da un gruppo degli Ateliers Vidéo - quello del foyer Gergovie, nel $14^{\mathrm{e}}$ arrondissement -, Les Aventuriers Sentimentaux, una commedia sui fantasmi e sulle illusioni dei rapporti di coppia tra Africa e Europa. Questo cortometraggio è un esempio interessante per mostrare in quale maniera la realizzazione debba negoziare fin dall'inizio con la ricezione prevista. Come lanciarsi in un progetto artistico senza orizzonte di attesa? Alla base, i criteri di scelta tematica dei nostri film sono: le voglie e le intenzioni del gruppo dei partecipanti ; il carattere attinente ma anche originale del nuovo lavoro in relazione alla nostra produzione precedente ; l'interesse ipotetico alle questioni suscitate da parte del pubblico (interno o esterno al foyer). Ora, nel frangente di questo film, i dibattiti avuti con i giovani ivoriani e maliani del gruppo si erano abbastanza rapidamente diretti verso il tema del divieto d'accesso alle donne nei foyers (eccetto durante le cerimonie e le visite del fine settimana) e del bisogno conseguente da parte dei ragazzi di incontrare delle ragazze all'esterno della residenza. Contando naturalmente tutte le difficoltà e le discrepanze linguistiche e culturali relative alla riuscita degli ipotetici incontri, per strada o altrove. Ne è derivata una docufiction molto divertente e spontanea, assolutamente non volgare o oltraggiosa, ma che si è finalizzata nell'ultima fase sapendo già che non avremmo potuto proiettarla all'interno di questo foyer. Era già successo una volta per il primo film degli ateliers di dover operare una "censura", evitando di filmare o di menzionare certi dettagli critici riguardanti un foyer, per non esporre la vergogna delle condizioni igieniche e abitative degli edifici ormai vetusti. Ma in questo caso, è stata la prima volta che abbiamo trattato un tema "vietato". Abitualmente abbiamo sempre preferito affrontare le questioni che valorizzassero i "buoni" sforzi dei sans papiers, quali l'apprendimento della lingua francese o la ricerca del lavoro : al seguito di una proiezione avvenuta un anno fa, l'imam di un foyer aveva citato uno dei nostri film per parlare di buoni esempi da seguire in fatto di approccio all'integrazione linguistica e professionale. Queste stesse preoccupazioni degli anziani e delle autorità del foyer, che, lo ricordiamo, sono come dei tutori e delle guide per i giovani che sono appena arrivati, non corrispondevano dunque alle cosiddette "distrazioni" mal considerate come quelle degli incontri amorosi al centro del film. Durante le riprese inoltre, avevamo dovuto domandare l'accordo per utilizzare una stanza e certe prime battute di un partecipante/ 
attore ivoriano esterno al foyer erano state giudicate fuori luogo dai residenti soninke maliani della stanza, rivelando così ugualmente determinati conflitti tra le diverse comunità e tra gli abitanti e gli "occupanti" esterni. Cosicché, alla fine, l'associazione ha assunto il suo ruolo di mediatrice, come dicevo, tra lo spazio del foyer et lo spazio della città, non solamente rigirando la sequenza discriminata, ma girando un nuovo film di "riparazione". Quest'ultimo (attualmente in montaggio), trattando ancora una volta di difficoltà di inserzione lavorativa, logistica e linguistica, potrà essere mostrato nel foyer Gergovie in questione, mentre il film "vietato", nato da altre esigenze dei giovani partecipanti, è stato già oggetto di proiezioni esterne e di dibattiti interessanti.

Figura 3. Proiezione al foyer Tolbiac (Ottobre 2017)

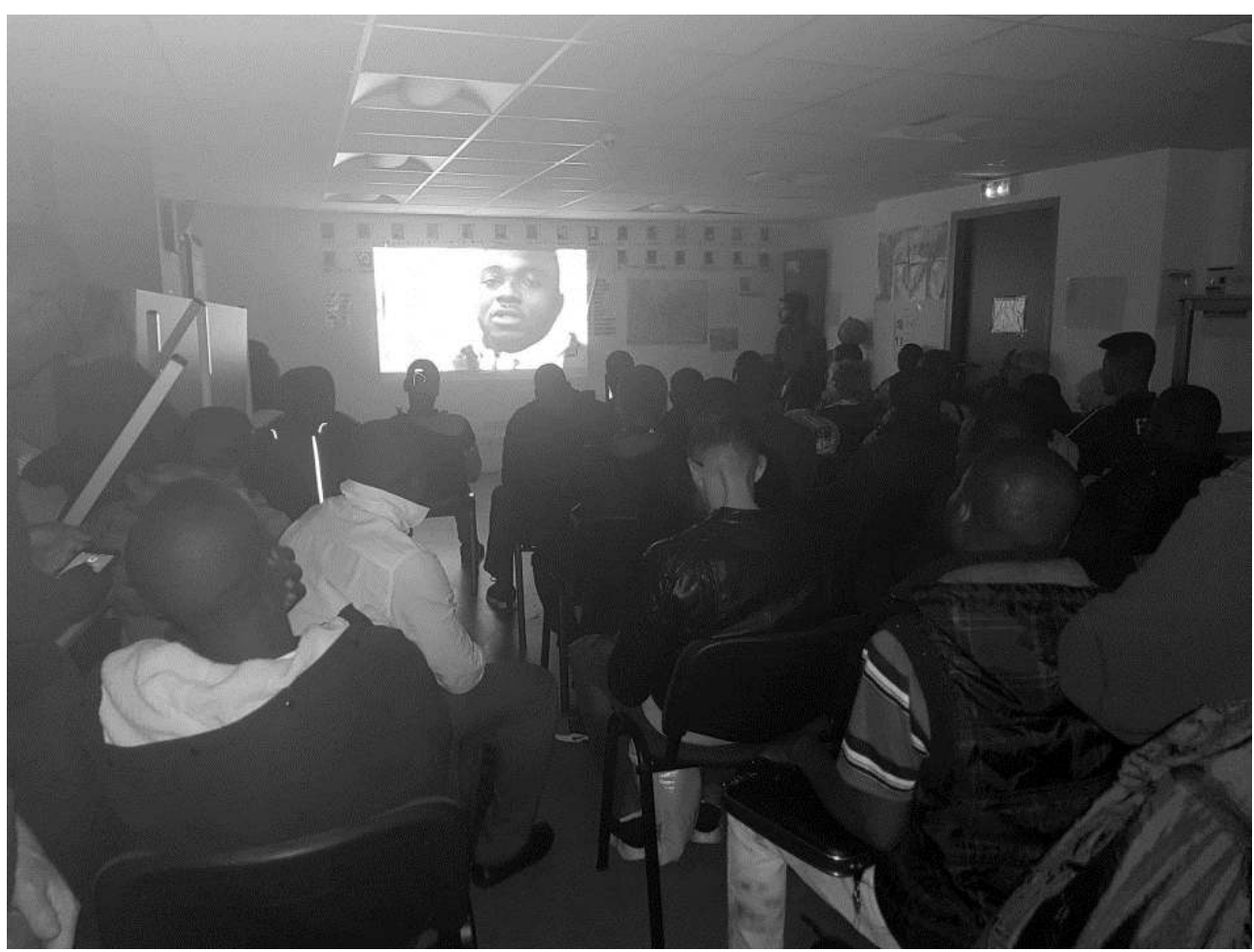

Le proiezioni sono un momento costitutivo fondamentale degli Ateliers Vidéo, poiché permettono ai partecipanti di presentare e di valorizzare loro stessi il lavoro svolto e le opere compiute, ma anche di poter vedere il film sul grande schermo in contesti inabituali e poter dibattere con il pubblico. Durante le proiezioni all'interno dei foyers, la situazione è simile, in piccolo, a quella descritta per il festival. Innanzitutto, si assiste a un via-vai continuo di gente: chi arriva in ritardo, chi parte in anticipo, chi esce per rispondere al cellulare che squilla. Il livello sonoro è abitualmente più elevato, comprendendo gli spostamenti di sedie, i commenti ad alta voce, le risate ad ogni sketch o gaffe o ad ogni apparizione di un amico nel film. Ci si identifica facilmente nella situazione rappresentata, si riconosce un insieme di non-detti che acquisiscono un'altra dimensione su un grande schermo, instaurando un'unica atmosfera di complicità. Arrivati alla discussione, la maggior parte delle domande è posta tipicamente dalle poche persone esterne al foyer, che hanno voglia di interpellare i partecipanti per informarsi sul dispositivo partecipativo usato, per fare commenti sulla musica, sulla lingua o sull'interpretazione degli attori. Alla fine, il momento conviviale delle bibite e degli snack 
chiude la serata con altre conversazioni, foto di gruppo, battute e prese di contatto per gli ateliers che verranno.

La ricezione si articola diversamente invece negli altri spazi della città, dove, al contrario dei foyers, la prevalenza di spettatori francesi sposta l'attenzione generale su altri aspetti del progetto. Nei mesi di Marzo e di Aprile abbiamo per esempio organizzato due proiezioni de Les Aventuriers Sentimentaux a Parigi, la prima in occasione di un seminario all'università - l'EHESS - e la seconda in una serata in un ristorante associativo di Belleville - il CIP20 -. I partecipanti si sono ritrovati per la prima volta immersi in contesti per loro sconosciuti, e al centro dell'interesse collettivo, seduti dietro una cattedra di un anfiteatro di una grande école, prendendo la parola al microfono e dialogando direttamente con delle persone non appartenenti all'ambiente del foyer. Il pubblico, a sua volta, ha molto apprezzato il film, mettendo in valore la sincerità e la spontaneità dei giovani, le dinamiche interculturali delle relazioni di coppia, il tono comico di diverse sequenze e il modo di giocare con certi fantasmi postcoloniali. Durante il dibattito, gli spettatori hanno posto domande sui percorsi migratori dei partecipanti, sui metodi di scrittura e di ripresa del film e sulla veridicità di alcune battute di dialogo riguardo il matrimonio, soggetto discusso alla fine del cortometraggio. I partecipanti hanno quindi fatto propria questa dimensione autobiografica, difendendo le idee espresse e raccontando le loro esperienze personali o spiegando altri elementi, come la lingua adottata, un "francese ivoriano" parlato ad Abidjan, che si distingue su vari fronti dal francese di Francia.

L'evento della proiezione, aprendo un nuovo spazio di ricezione, costituisce così una maniera non solo di partecipare attivamente nello spazio pubblico, di presentare il proprio lavoro, di costruire un'immagine di sé diversa, di iscriversi in un ambiente più ampio ed eterogeneo, ma anche di raccogliere infine qualche gratificazione - farsi applaudire dal pubblico, vedere il proprio nome sui titoli di coda, farsi fermare alla fine della proiezione per altre domande -. La partecipazione agli ateliers, se vissuta in questo modo (in dirittura d'arrivo non sono molti gli assidui), può dunque articolare creazione e ricezione, conoscenza e scambio, adempiendo probabilmente agli obiettivi prefissi dalle politiche pubbliche sull'animazione culturale e, si spera soprattutto, alle aspettative concrete dei partecipanti nei loro progetti di vita a Parigi.

\section{Conclusione}

14 Il breve resoconto analitico esposto in questo articolo dimostra in tal modo le negoziazioni necessarie al fine di produrre e diffondere i film associativi autobiografici dei migranti nel tentativo di iscrivere gli spazi e le identità dei foyers in quelli del quartiere e della città. Adattamenti e soluzioni che cercano di rispondere allo stesso tempo alle esigenze dei residenti e a quelle delle autorità dei foyers, alle domande delle istituzioni parigine che appoggiano e seguono il progetto, a un metodo di "pedagogia militante" e alle curiosità e agli interessi degli altri luoghi e degli altri pubblici della città. Ciò che mette tutti d'accordo sembra in ogni caso l'occasione di scambio sinceramente umana e orizzontale che gli Ateliers Vidéo offrono, così come la maniera più soggettiva, partecipativa e leggera con cui le nostre narrazioni autobiografiche affrontano i diversi aspetti problematici della migrazione, a differenza delle rappresentazioni comuni, monodirezionali e tragiche con le quali i media e i cinema occidentali hanno abituato il loro pubblico. 
BIBLIOGRAFIA

\section{Riferimenti bibliografici}

ADEL/ALFA Consultants, éds., 1999, Les foyers dans la ville. Foyers des travailleurs migrants et politique de la ville, Paris, FAS.

Bouamama S., 2002, «Les discours de l'interculturalité : modèles, enjeux et contradictions ", in Jovelin E., dir., Le travail social face à l'interculturalité. Comprendre la différence dans les pratiques d'accompagnement social, Paris, L'Harmattan, pp. 31-42.

Boucher M., 2006, « Le nouvel espace de contrôle social en France. Travailleurs sociaux et médiateurs sociaux à l'épreuve des logiques d'intégration, de régulation et de racisation », in Prieur E., Jovelin E., Blanc M., dir., Travail social et immigration. Interculturalité et pratiques professionnelles, Paris, L'Harmattan, pp. 209-222.

Jovelin E., 2002, « Comprendre l'interculturalité : l'ouverture à l'autre », in Jovelin E., dir., Le travail social face à l'interculturalité. Comprendre la différence dans les pratiques d'accompagnement social, Paris, L'Harmattan, pp. 17-30.

Legault G., 2006, « Interculturalité et diversité des pratiques professionnelles. L'étranger, cet autre moi-même », in Prieur E., Jovelin E., Blanc M., dir., 2006, Travail social et immigration. Interculturalité et pratiques professionnelles, Paris, L'Harmattan, pp. 297-304.

Morel D., 2002, "Vers une "relative proximité" : la distance, une notion centrale commune à l'épistémologie et à l'éthique ", in Jovelin E., dir., Le travail social face à l'interculturalité. Comprendre la différence dans les pratiques d'accompagnement social, Paris, L'Harmattan, pp. 287-294.

Piault M. H., 2000, Anthropologie et cinéma. Passage à l'image, passage par l'image, Paris, Teraedre.

Wodiczko K., 2016a, « I want to be a Catalyst. An Interview with William Furlong, 1988 », in Wodiczko K., dir., Transformative Avant-Garde and Other Writings, London, Black and Dog Publishing, pp. 184-186.

Wodiczko K., 2016b, « The Inner Public, 2015 », in Wodiczko K., dir., Transformative Avant-Garde and Other Writings, London, Black and Dog Publishing, pp. 287-299.

\section{NOTE}

1. ADEL/ALFA Consultants, 1999 : 50 . Tutte le traduzioni dal francese e dall'inglese sono di mia mano.

2. Boucher, $2006: 219$.

3. Legault, $2006: 301$

4. Vedi l'antropologia condivisa proposta da Piault, $2000: 237$.

5. Morel, $2002:$ 287-294.

6. Jovelin, $2002: 26$.

7. Bouamama, $2002: 35$.

8. Wodiczko, 2016b : 288-289. 


\section{RIASSUNTI}

Gli Ateliers Video, condotti dall'associazione Parcours a Parigi, formano da due anni e mezzo i residenti dei foyers dei lavoratori migranti d'Ile-de-France alla creazione audiovisiva. L'articolo, che si presenta come un resoconto analitico delle attività svoltesi nell'ambito di questa associazione, mette in risalto la questione della ricezione a partire delle dinamiche legate al rapporto tra lo spazio interno dei foyers e lo spazio esterno della città. L'analisi verte sulle maniere in cui l'organizzazione di un festival cinematografico, il funzionamento degli ateliers, le riprese dei film e la loro ricezione, dipendono fortemente da numerosi fattori quali la regolamentazione dei foyers, la trasmissione culturale tra gli abitanti delle diverse generazioni, la convivenza tra le etnie presenti e la loro relazione con gli altri abitanti del quartiere e della città.

I cortometraggi dell'associazione, attraverso un metodo partecipativo durante il quale tutti i partecipanti contribuiscono alle tappe progressive della realizzazione, mettono in scena le storie autobiografiche dei migranti sans papiers, spesso unendo documentario e finzione. I partecipanti, in maggioranza provenienti dal Mali, ma anche dalla Costa d'Avorio, Mauritania, Senegal, Algeria, Capo Verde, diventano per la prima volta allo stesso tempo spettatori di film (delle proiezioni sono organizzate lungo tutto il semestre) e creatori (sceneggiatori, registi, attori, tecnici, montatori). Nel contesto particolare dei foyers dei lavoratori migranti, l'articolo sottolinea la negoziazione necessaria tra la produzione e la ricezione delle immagini, tra un "Sud" africano riprodotto negli alloggi sociali e un "Nord" parigino sempre presente tramite le politiche cittadine, i finanziamenti pubblici e i luoghi di diffusione e dibattito.

Residenze ; Parigi ; immigrazione africana ; video ; partecipativo ; pedagogia ; proiezioni

Les Ateliers Vidéo, menés au sein de l'association Parcours à Paris, forment depuis deux ans et demi les résidents des foyers des travailleurs migrants d'Ile-de-France à la création audiovisuelle. L'article, qui se présente comme un compte-rendu analytique des activités déroulées dans le cadre de cette association, met en avant la question de la réception à partir des dynamiques liées au rapport entre l'espace interne des foyers et l'espace externe de la ville. L'analyse porte sur les manières dont l'organisation d'un festival de cinéma, le fonctionnement des ateliers, le tournage des films et leur réception dépendent fortement de plusieurs facteurs tels que la réglementation des foyers, la transmission culturelle entre les habitants de différentes générations, la cohabitation entre les ethnies présentes et la relation de celles-ci avec les autres habitants du quartier et de la ville.

Les court-métrages de l'association, à travers une démarche participative durant laquelle tous les participants contribuent aux étapes progressives de la réalisation, mettent en forme les récits autobiographiques des migrants sans papiers, souvent mélangeant documentaire et fiction. Les participants, en majorité provenant du Mali, mais aussi Côte d'Ivoire, Mauritanie, Sénégal, Algérie, Cap-Vert, deviennent pour la première fois en même temps des spectateurs de films (des projections sont organisées tout au long du semestre) et des créateurs (scénaristes, réalisateurs, acteurs, techniciens, monteurs). Dans le contexte particulier des foyers des travailleurs migrants, l'article souligne la négociation nécessaire entre la production et la réception des images, entre 
un « Sud » africain reproduit dans les logements sociaux et un « Nord » parisien toujours présent à travers les politiques de la ville, les financements publiques et les lieux de diffusion et débat.

INDICE

Mots-clés : Foyers ; Paris ; immigration africaine ; vidéo ; participatif ; pédagogie ; projections

\section{AUTORE}

\section{GUGLIELMO SCAFIRIMUTO}

Doctorant en Études Cinématographiques à l'Université Sorbonne Nouvelle - Paris 3, LIRA Laboratoire International de Recherche en Arts. 